\title{
A comparison and synthesis of rehabilitation definitions used by consumers (Google), major Stakeholders (survey) and researchers (Cochrane Systematic Reviews): a terminological analysis
}

\author{
Chiara ARIENTI 1, Michele PATRINI 1, *, Alex POLLOCK ${ }^{2}$, \\ Stefano G. LAZZARINI ${ }^{1}$, Aydan ORAL ${ }^{3}$, Stefano NEGRINI 4, 5
}

${ }^{1}$ IRCCS Fondazione Don Carlo Gnocchi, Milan, Italy; ${ }^{2}$ Nursing, Midwifery and Allied Health Professions Research Unit, Glasgow Caledonian University, Glasgow, UK; 32Department of Physical Medicine and Rehabilitation, Istanbul Faculty of Medicine, Istanbul University, Istanbul, Turkey; ${ }^{4}$ Department of Biomedical, Surgical and Dental Sciences, University "La Statale", Milan, Italy; 5 IRCCS Istituto Ortopedico Galeazzi, Milan, Italy

*Corresponding author: Michele Patrini, IRCCS Fondazione Don Carlo Gnocchi, Milan, Italy. E-mail: mpatrini@dongnocchi.it

\section{A B S T R A C T}

BACKGROUND: The term "rehabilitation" is heterogeneously used in the health context. Different interpretations can lead to disagreements, misunderstandings and different interpretations of what rehabilitation is between who provides it, who receives it and who studies it. The aim of this study was to conduct a terminological analysis of the different rehabilitation definitions used by different audiences: consumers, rehabilitation stakeholders and researchers.

METHODS: We performed a terminological analysis with comparison of three different collections of rehabilitation definitions in English language. We performed: systematic reviews of databases representing consumers and lay persons (Google) and researchers (Cochrane Systematic Reviews [CSRs]), and a survey of rehabilitation stakeholders (Cochrane Rehabilitation Advisory Board). To aggregate words that had the same underlying concepts, their roots were extracted, and their occurrences counted. The 30 most frequent roots of each search were included. The 3 obtained collections were compared and similarities calculated. An overall collection of the most important 30 roots was obtained weighting those obtained in each single collection. All analyses have been performed using Excel.

RESULTS: One hundred and eighty-seven rehabilitation definitions were identified: 23 from CSRs, 36 from the survey and 128 from Google. The most frequent roots were "function*" (92\%), followed by "proces*” (69\%o), "health*” (59\%), "disab*" (53\%o), and "person*” (50\%0). The most common relevant roots related to rehabilitation concept were "proces*" (73\%) in Google, "function*" (109\%) in the survey and "disab*" (41\%) in CSRs. The noun "function" prevailed in Google and "functioning" in the survey.

CONCLUSIONS: According to our findings, any definition of rehabilitation for research purposes should include the identified terms, focusing on the concept of process and considering the main elements of functioning (and function), disability, person, health, optimization and environment.

(Cite this article as: Arienti C, Patrini M, Pollock A, Lazzarini SG, Oral A, Negrini S. A comparison and synthesis of rehabilitation definitions used by consumers (Google), major Stakeholders (survey) and researchers (Cochrane Systematic Reviews): a terminological analysis. Eur J Phys Rehabil Med 2020;56:682-9. DOI: 10.23736/S1973-9087.20.06583-1)

KEY WORDS: Rehabilitation; Terminology; Surveys and Questionnaires.

$\mathrm{T}$ he term "rehabilitation" is heterogeneously used in many different contexts, such as health, education, law and engineering. Even in the context of health, different interpretations of rehabilitation can lead to disagreements and misunderstandings between who provides it, who receives it and who studies it. ${ }^{1}$ Searching through dictionar- 


\section{COPYRIGHT $^{\circledR} 2020$ EDIZIONI MINERVA MEDICA}

ies or the internet does not provide much help, as most definitions are imprecise, lack details and are not based on evidence. ${ }^{2}$ The currently available definitions of rehabilitation appear to fall short on their purpose of exactly defining what we need for our scientific purposes, ${ }^{1}$ and it is hard to deduce the inclusion and exclusion criteria of what is rehabilitation. ${ }^{3}$

As part of its activities, Cochrane Rehabilitation identified three specific situations that provided a challenge to the meaning of the term "rehabilitation". The first instance was on whether to define balneotherapy ${ }^{4}$ and acupuncture ${ }^{5}$ as rehabilitation interventions or not. The second was concerning a Cochrane Systematic Review (CSR) where the authors of the paper used the term "Penile Rehabilitation" despite their focus was on drugs only. ${ }^{6}$ The third situation was related to whether or not to include some medical treatments (such and spasmolytic drugs or nonsteroidal anti-inflammatory drugs) in the World Health Organization Package of Interventions for Rehabilitation ${ }^{7}$ for conditions such as rheumatoid arthritis and spinal cord injury.

We thought that an empirical investigation of rehabilitation definitions in the health context coming from different sources might help clarify the matter. The aim of this study was to conduct a terminological analysis of the different rehabilitation definitions used by three different, yet vital, audiences: consumers, rehabilitation stakeholders and researchers. We wanted to identify the most frequent concepts used to describe rehabilitation and, in doing so, providing key elements to be considered in developing a new rehabilitation definition for scientific purposes.

\section{Methods}

The methodology includes a terminological analysis and comparison of three different collections of rehabilitation definitions representing three different perspectives: 1) a Google search to explore definitions used primarily by consumers and lay persons; 2) a survey administered to Cochrane Rehabilitation stakeholders; 3 ) the researchers' perspective coming from CSRs, with a specific focus on stroke CSRs.

\section{Google search collection}

An extensive search in Google was performed on January $4^{\text {th }}, 2020$ to collect the most used definitions of rehabilitation worldwide. To avoid Google Algorithms filtering results, proxy servers were used from five English speaking countries in different continents: Australia, India, South
Africa, United Kingdom and USA. In order to represent South America as well, we included its biggest and most populated country, Brazil. The two search strings were: 1) "rehabilitation definition"; 2) "what is the definition of rehabilitation?". They were used quoted and unquoted by a single author (MP). Results have been obtained with the automatic duplicate removal function of Google activated and deactivated. For each search, the first 200 results were collected with a JavaScript script specifically coded for the task. The exclusion criteria were: no definition reported, definition of anything that is not related to humans (such as land, building or animals), definition of specific rehabilitation professions and definition relating to rehabilitation of offenders or of drugs/alcohol abusers. Google Books entries were also removed from results due to the difficulties in retrieving the full texts and the impossibility of automatically removing the duplicates.

\section{Cochrane Rehabilitation stakeholders survey collection}

A survey was performed to collect the current definitions used by the major rehabilitation stakeholders as represented in the Advisory Board of Cochrane Rehabilitation. ${ }^{8}$ This Board comprises 35 members representing 14 world and continental scientific societies, 12 major scientific journals, several low- and middle-income countries, consumers, Cochrane groups and rehabilitation experts. We also included the 10 members of Cochrane Rehabilitation Executive Committee ${ }^{9}$ and the 22 participants invited to the $3^{\text {rd }}$ Cochrane Rehabilitation Methodology Meeting about "Rehabilitation Definition for research purposes," where this paper was thoroughly discussed.

The survey was sent out to a total of 55 participants on November $20^{\text {th }}, 2019$ and included a series of open questions. The questions asked for specific definitions used by their organization and organizations/bodies known to them, preferences relating to definitions, terms and expressions, as well as the scope of the interventions delivered by rehabilitation professionals. Three reminders were sent, and data collection was concluded on January 17th, 2020.

\section{Cochrane Systematic Reviews collection}

The search was performed on $5^{\text {th }}$ February 2020 in PubMed database using the following search string: ["Cochrane Database Syst Rev" [jour] AND ("rehabilitation"[TIAB])] until January $31^{\text {st }}, 2020$. This was in accordance with the selection criteria previously published by the Cochrane Rehabilitation Review Committee. ${ }^{10}$ Since we were interested in the definitions of rehabilitation and not in the single intervention provided, we identified the CSRs that 


\section{COPYRIGHT $^{\circledR} 2020$ EDIZIONI MINERVA MEDICA}

ARIENTI

TERMINOLOGICAL ANALYSIS OF REHABILITATION DEFINITIONS

had the term rehabilitation in the title or in the abstract. Then, we collected all the descriptions of rehabilitation inside each CSR.

We also collected a second group of CSRs, including exclusively stroke CSRs on the effectiveness of rehabilitation interventions, which represents the biggest group of CSRs in the rehabilitation field alongside those on back pain. ${ }^{11}$ We performed the search on December 11th 2019 using the DORIS (Database of Research In Stroke) database and including any CSR on interventions categorized as "occupational therapy," "physiotherapy," "psychological therapy," "speech therapy," "visual" or "complementary medical therapy" (i.e. not CSRs categorized only as "diet", "nursing", "pharmacology," "radiology," "radiotherapy," "service provision" or "surgery"). CSRs were categorized according to the participants, intervention and outcomes defined within their title, and full texts of relevant CSRs were explored.

\section{Data analysis}

A word frequency count was obtained from each of the three collections of definitions (Google, stakeholders survey, and CSRs) and from a fourth built merging all of them. The count was performed using a free online word cloud generator (https://www.wordclouds.com) after excluding articles and propositions. To aggregate words that had the same underlying concept, the roots of the words (e.g. "disab*" to include all terms like disable, disability, etc.) were extracted and their occurrences were counted. In some cases, this approach was not applicable as it would have led to a loss of information. For example, the words "multidimensional," "multidisciplinary" and "multiple" have the common root "multi" but convey different meanings. In such cases, the words were reduced to their singular form and counted. Lastly, the words that could not be aggregated with others were kept unmodified.

To create a collection summarizing the results obtained in each one (ALL), we arbitrarily decided to include the 30 most frequent roots derived from each collection, including the following steps. First, the frequency of the occurrence of the roots was calculated for each list providing the per thousand distribution of the root occurrence. This allowed us to adjust for the different sizes of each list. Second, the collections were merged into a single collection providing a list of all the included unique roots whose value was calculated as an average of the respective per thousand value in each list.

To compare the three original collections using the same terms, three new collections have been created out of the three original ones by only selecting the 30 most frequent roots present in ALL, and we used the average frequency of the occurrence of the roots obtained as reported above.

To check for the possibility that definitions could include each single root more than once, we also counted the occurrence in each single definition of all the roots with a frequency of at least 5\% in ALL. Finally, the root "function*" has been further analyzed counting the frequency of the two nouns "functioning(s)" and "function(s)," since both are contained in the same root but have a different meaning in rehabilitation. All analyses have been performed using Excel.

For the Cochrane Stroke CSRs, we extracted data on: 1) explicit definition of rehabilitation (from the background or methods section); 2) description/definition of intervention (from the 'Types of interventions' section within criteria for considering studies for this CSR). The extracted data was explored to identify similarities and differences across CSRs and analyzed narratively.

The lists of all included definitions and collections of roots are available as Supplementary Digital Material 1 (Supplementary Table I, Supplementary Table II, Supplementary Table III, Supplementary Table IV).

\section{Results}

Seventy-six percent of participating stakeholders answered the survey. Most of them were Physical and Rehabilitation Medicine (PRM) physicians (64\%); and thirty-six definitions were provided for rehabilitation in health (mandatory question) and rehabilitation in general (optional question).

From the Google search, 6928 links were retrieved, and after duplicates removal, a total of 1240 websites were screened. The exclusion criteria were then applied and resulted in finding 243 definitions. From those, 128 unique definitions were identified.

After screening 434 CSRs, 223 CSRs were found to be relevant to rehabilitation, but only 93 (42\%) papers contained the term rehabilitation in the title and/or abstract (Figure 1). Of these, 13 (14\%) CSRs provided a description (definition) of rehabilitation. Twenty-eight (30\%) considered a specific intervention as rehabilitation, like cognitive rehabilitation $(14 \%)$, cardiac rehabilitation $(14 \%)$, vocational rehabilitation (3\%), physical rehabilitation, physical training and occupational therapy (1\%). Fifty-two CSRs $(56 \%)$ did not provide any explicit description of rehabilitation or were identified with a specific intervention. The term was only present in the title or abstract without any contextualization. 


\section{COPYRIGHT ${ }^{\odot} 2020$ EDIZIONI MINERVA MEDICA}

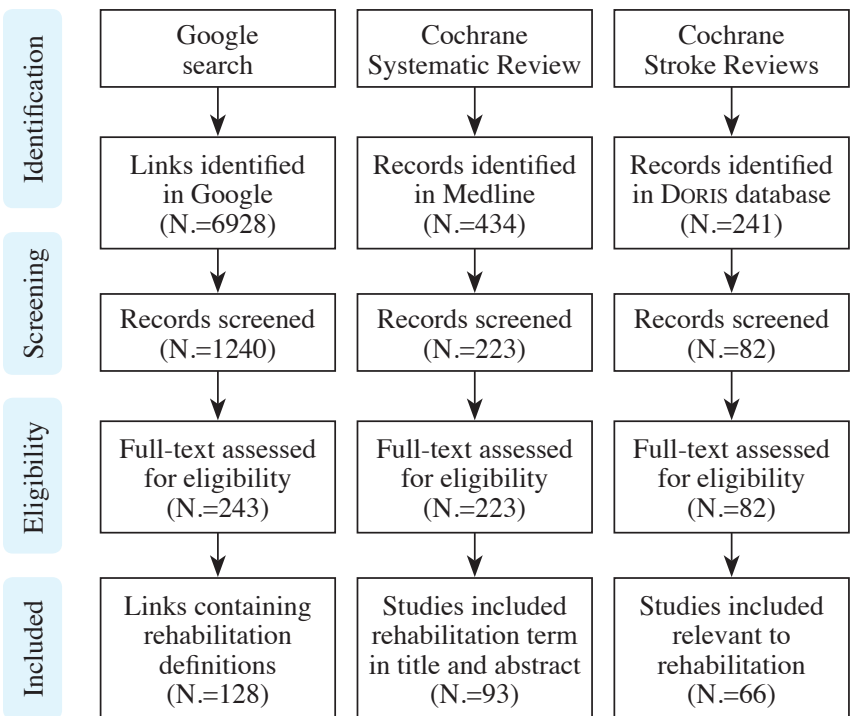

Figure 1.-Flow-chart of the study.

Similar results were found in Cochrane Stroke CSRs. Indeed, based on the intervention categorization of 241 Cochrane Stroke CSRs, the full text of 82 were considered, and 66 were judged to be directly relevant to rehabilitation. Of these, 25 focused on any intervention or a broad group of interventions for a specific problem, 19 focused on a named intervention for 'general' (or nonspecified) outcomes following stroke and 22 focused on a named intervention for a specific population or outcome/ problem after stroke. None of the 66 CSRs provided an explicit definition of rehabilitation. When describing or categorizing rehabilitation interventions, 7 CSRs referred to some or all of the terms "restitution," "compensation" and "substitution" (or their derivatives), and 3 referred to the International Classification of Functioning, Disability and Health (ICF). It was noted that the term rehabilitation was commonly used in combination with a term relating to the specific outcome or problem of interest (e.g. "memory rehabilitation," "upper limb rehabilitation") and that definitions provided in the CSRs tended to focus on the outcome or problem of interest, rather than the concept of rehabilitation.

\section{Terminological analysis}

One hundred and eighty-seven single rehabilitation definitions were identified. The lists of words obtained from the definitions retrieved from Google search, survey results and CSRs have been displayed in three tag clouds (Figure 2 ). The terminological analysis of all rehabilitation definitions showed 58 unique roots, combining the 30 most frequent roots of the three collections.

The survey collection compared with Google and CSRs collections had more similarities $(50 \%$ and $47 \%$ respectively) respect of the comparison between CSRs and Google (33\%). The most frequent roots related to rehabilitation were "function*" (92\%), followed by "proces*" (69\%o) and "disab*" (59\%o), and "health*" and "person*" (53\% and 50\% respectively). We also found other relevant rehabilitation roots like "individual*" (42\%), "improv*" and "particip*" (38\%), and "optim*" (24\%o) (Table I). Looking for the same roots in each collection, "proces*" (73\%o) in Google, and "function*" (109\%) in the survey were the most common in absolute, while in

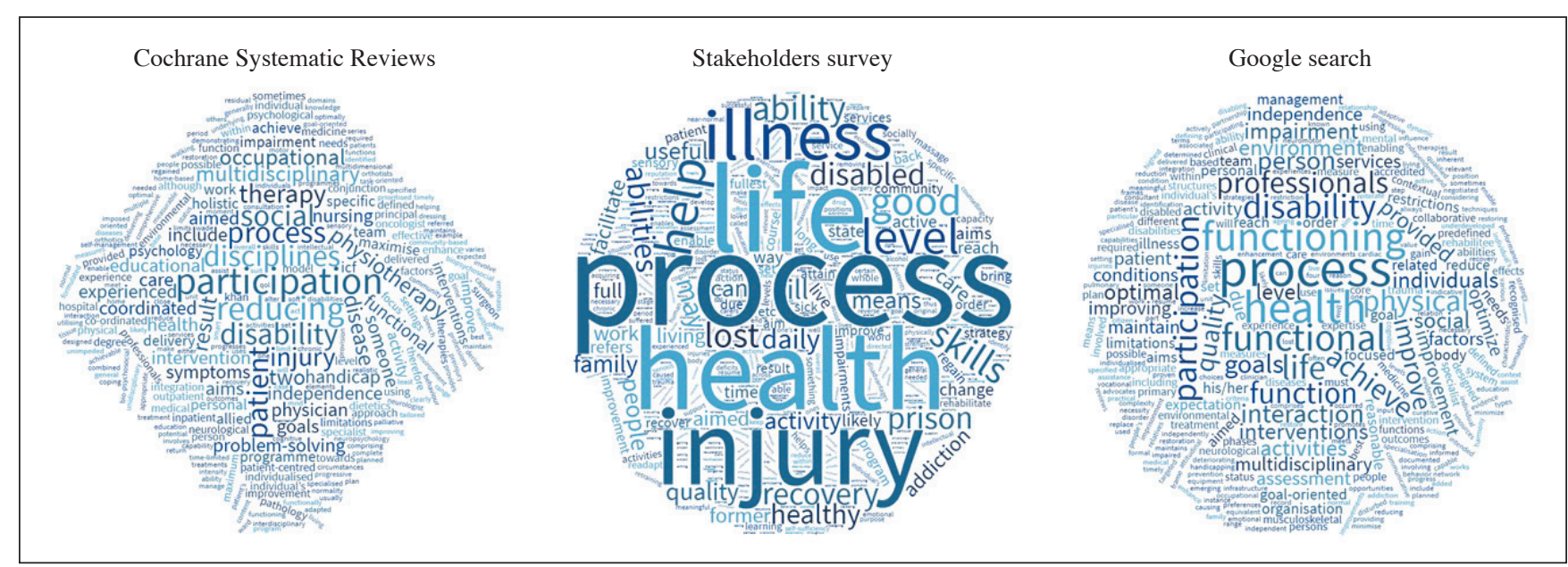

Figure 2.-Wordclouds of the definitions retrieved from Cochrane Systematic Reviews, Stakeholders survey, Google Search. 


\section{COPYRIGHT ${ }^{\circledR} 2020$ EDIZIONI MINERVA MEDICA}

ARIENTI

TERMINOLOGICAL ANALYSIS OF REHABILITATION DEFINITIONS

TABLE I.-Roots frequency in "all” and each single collection.

\begin{tabular}{|c|c|c|c|c|c|c|c|c|c|c|}
\hline \multicolumn{2}{|c|}{ ALL } & \multicolumn{3}{|c|}{ Cochrane Systematic Reviews } & \multicolumn{3}{|c|}{ Stakeholders survey } & \multicolumn{3}{|c|}{ Google search } \\
\hline Root & $\%$ & Root & Count & $\%$ & Root & Count & $\%$ & Root & Count & $\%$ \\
\hline function* & 92 & patient* & 11 & 56 & function* & 29 & 109 & proces* & 59 & 73 \\
\hline proces* & 69 & particip* & 10 & 51 & improv* & 15 & 56 & function* & 53 & 66 \\
\hline disab* & 59 & reduc* & 10 & 51 & proces* & 14 & 53 & restor* & 44 & 55 \\
\hline health* & 53 & aim* & 8 & 41 & disab* & 13 & 49 & health* & 41 & 51 \\
\hline person* & 50 & disab* & 8 & 41 & health* & 13 & 49 & person* & 41 & 51 \\
\hline individual* & 42 & function* & 8 & 41 & acti* & 12 & 45 & condition* & 38 & 47 \\
\hline soci* & 42 & therap* & 8 & 41 & goal* & 12 & 45 & disab* & 37 & 46 \\
\hline aim* & 38 & discipline* & 7 & 36 & person* & 11 & 41 & life* & 37 & 46 \\
\hline improv* & 38 & goal* & 7 & 36 & individual* & 10 & 38 & injur* & 35 & 44 \\
\hline particip* & 38 & individual* & 7 & 36 & optim* & 9 & 34 & soci* & 34 & 42 \\
\hline goal* $^{*}$ & 36 & intervention* & 7 & 36 & particip* & 9 & 34 & help* & 32 & 40 \\
\hline life* & 31 & proces* & 7 & 36 & environment* & 8 & 30 & ill* & 32 & 40 \\
\hline patient* & 34 & soci* & 7 & 36 & life* & 8 & 30 & normal* & 27 & 34 \\
\hline injur* & 32 & coordinat* & 6 & 31 & achiev* & 7 & 26 & physical* & 27 & 34 \\
\hline acti* & 30 & deliver* & 6 & 31 & aim* & 7 & 26 & abil* & 22 & 27 \\
\hline reduc* & 32 & disease* & 6 & 31 & independe* & 7 & 26 & acti* & 21 & 26 \\
\hline condition* & 27 & experienc* & 6 & 31 & intervention* & 7 & 26 & treat* & 20 & 25 \\
\hline intervention* & 28 & improv* & 6 & 31 & physical* $^{*}$ & 7 & 26 & individual* & 19 & 24 \\
\hline physical* & 25 & injur* & 6 & 31 & profession* & 7 & 26 & optim* & 19 & 24 \\
\hline optim* & 24 & education* & 5 & 26 & impair* & 6 & 23 & achiev* & 17 & 21 \\
\hline restor* & 22 & health* & 5 & 26 & interact* & 6 & 23 & disease* & 17 & 21 \\
\hline disease* & 23 & $\max ^{*}$ & 5 & 26 & level* & 6 & 23 & level* & 17 & 21 \\
\hline achiev* & 20 & multidisciplin* & 5 & 26 & provi* & 6 & 23 & aim* & 16 & 20 \\
\hline level* & 18 & occupational & 5 & 26 & quality & 6 & 23 & possible & 16 & 20 \\
\hline therap* & 19 & person* & 5 & 26 & relat* & 6 & 23 & mental* & 15 & 19 \\
\hline help* & 16 & physiotherapy & 5 & 26 & condition* & 5 & 19 & recover* & 15 & 19 \\
\hline ill* & 16 & program* & 5 & 26 & patient* & 5 & 19 & someone* & 15 & 19 \\
\hline discipline* & 17 & psychol* & 5 & 26 & reduc* & 5 & 19 & liv* & 13 & 16 \\
\hline normal* & 14 & result & 5 & 26 & soci* & 5 & 19 & medic* & 13 & 16 \\
\hline coordinat* & 14 & two & 5 & 26 & use* & 5 & 19 & enabl* & 12 & 15 \\
\hline \multicolumn{11}{|c|}{ The "all" collection has been calculated weighting the contribution of each single collection. } \\
\hline \multicolumn{11}{|c|}{ ABLE II.-Number of definitions including at least once the most frequent roots (at least $5 \%$ of occurrence) in each collection. } \\
\hline & \multicolumn{3}{|c|}{$\begin{array}{c}\text { Cochrane } \\
\text { Systematic Reviews (23 definitions) }\end{array}$} & \multicolumn{4}{|c|}{ Stakeholder survey (36 definitions) } & \multicolumn{3}{|c|}{ Google search (128 definitions) } \\
\hline Root & \multicolumn{2}{|c|}{ Count } & \multicolumn{2}{|l|}{$\%$} & Count & \multicolumn{2}{|l|}{$\%$} & Count & \multicolumn{2}{|c|}{$\%$} \\
\hline function* & \multicolumn{2}{|c|}{7} & \multicolumn{2}{|l|}{$30 \%$} & 24 & \multicolumn{2}{|l|}{$67 \%$} & 49 & \multicolumn{2}{|c|}{$38 \%$} \\
\hline proces* & \multicolumn{2}{|c|}{7} & \multicolumn{2}{|l|}{$30 \%$} & 12 & \multicolumn{2}{|l|}{$33 \%$} & 49 & \multicolumn{2}{|c|}{$38 \%$} \\
\hline disab* & \multicolumn{2}{|c|}{7} & \multicolumn{2}{|l|}{$30 \%$} & 10 & $28 \%$ & & 29 & & \\
\hline health* & & 5 & $22 \%$ & & 13 & $36 \%$ & & 36 & & \\
\hline person* & & 5 & $22 \%$ & & 10 & $28 \%$ & & 34 & & \\
\hline
\end{tabular}

TABLE III.-Presence of nouns "function(s)" and "functioning(s)" in the collections. The distinction has been made since both nouns are contained in the root "function*."

\begin{tabular}{|c|c|c|c|c|c|c|c|}
\hline \multirow[t]{2}{*}{ Nouns } & \multicolumn{2}{|c|}{$\begin{array}{c}\text { Cochrane } \\
\text { Systematic Reviews }\end{array}$} & \multicolumn{2}{|c|}{$\begin{array}{l}\text { Stakeholders } \\
\text { Survey }\end{array}$} & \multicolumn{2}{|c|}{$\begin{array}{l}\text { Google } \\
\text { Search }\end{array}$} & \multirow{2}{*}{$\begin{array}{c}\text { Total } \\
\% 0\end{array}$} \\
\hline & Count & $\%$ & Count & $\%$ & Count & $\%$ & \\
\hline Function(s) & 2 & $4 \%$ & 8 & $7 \%$ & 20 & $11 \%$ & $7 \%$ \\
\hline Functioning & 1 & $2 \%$ & 10 & $8 \%$ & 15 & $8 \%$ & $6 \%$ \\
\hline
\end{tabular}




\section{COPYRIGHT $^{\circledR} 2020$ EDIZIONI MINERVA MEDICA}

TERMINOLOGICAL ANALYSIS OF REHABILITATION DEFINITIONS

ARIENTI

TABLE IV.-Percentage of definitions including the nouns "function(s)" and "functioning(s)" in each collection.

\begin{tabular}{lccc}
\hline Nouns & $\begin{array}{c}\text { Cochrane } \\
\text { Systematic Reviews } \\
\text { (23 definitions) }\end{array}$ & $\begin{array}{c}\text { Stakeholder survey } \\
\text { (36 definitions) }\end{array}$ & $\begin{array}{c}\text { Google search } \\
\text { (128 definitions) }\end{array}$ \\
\hline Function(s) & $9 \%$ & $22 \%$ & $16 \%$ \\
Functioning & $4 \%$ & $42 \%$ & $8 \%$ \\
\hline
\end{tabular}

CSRs the most common was "disab*" (41\%), but following "patient*" (56\%), and "particip*" and reduc*" $(51 \%)$.

When looking at the percentage of definitions including the 5 words whose overall frequency was above $5 \%$, "function*" was by far the most frequent in the survey (68\%), while in Google and CSRs it was as frequent as "proces*" (38\% and 30\%, respectively) and "disab*" in CSRs only (Table II). Finally, the noun "functioning(s)" was more frequent than "function(s)" in the survey only, and that is in both the collections (Table III) and definitions (Table IV).

\section{Discussion}

The novelty of the study was to identify the concepts most frequently used to describe rehabilitation from three different sources: a Google search to mostly represent consumers, a survey from Cochrane Rehabilitation stakeholders, and CSRs, with a specific focus on stroke, to represent the perspective of researchers. We found that the different perspectives are reflected in the terminology used in definitions, with researchers (CSRs) differing from the others and stakeholders focusing on functioning versus function used by all the others.

The findings show that most of CSRs do not present a definition of rehabilitation and that $30 \%$ consider rehabilitation as a specific intervention. In particular, Cochrane Stroke CSRs focused on any intervention or a broad group of interventions for a specific problem and/or population, and non-specified outcomes. The main specific intervention described was cardiac rehabilitation, defined by the British Association for Cardiovascular Prevention and Rehabilitation"12 as a "sum of activities to provide the best possible physical, mental and social conditions, so that the patients may preserve or resume optimal functioning." This definition emphasizes the exercise training programs as a central component of cardiac rehabilitation. ${ }^{13}$

The most important WHO classification related to rehabilitation is the "International Classification of Functioning, Disability and Health" (ICF): 14 coherently, these terms are the most reported, after the most frequent root "proces*" and immediately before "person*." Nevertheless, there are important differences among audiences, mostly coming from the verbs used in conjunction with these names: "improv*" for stakeholders, "restor*" for consumers, and "reduc*" and "aim*" for researchers. The first seem to focus mostly on the positive attitude of improving the existing residual capacity, while the others concentrate on reducing the damage. This is highly coherent with the philosophical and ethical principles of ICF compared to the previous classification ICIDH: ${ }^{15}$ persons are defined by who they are and not by what they miss. The influence of the ICF on stakeholders mostly is clearly shown by the striking difference in the use of the word "functioning" vs "function."

The terminological analysis of all collections highlights the most frequently used roots relevant to rehabilitation, which are: "proces*," "function*," "reduc*," "health*," "disab*" and "person*" with a different distribution and meaning according to the three different perspectives. The root "proces*" was the most common in Google, where the rehabilitation definitions are often derived from dictionaries that, usually, use a concise form for the definitions to facilitate the understanding of consumers. Furthermore, "proces*" is commonly defined as "a series of actions that we take in order to achieve a result", 16 that might represent the consumers' perspective.

"Function*" was the more frequent root resulted from the survey, both when counted together and separately from "functioning(s)." These terms have been developed especially for the rehabilitation field, ${ }^{17}$ and they have been codified in the ICF classification. ${ }^{14,18}$ These terms represent Cochrane Rehabilitation stakeholders' perspective.

Finally, "reduc*" and "disab*" were the most frequent roots provided in CSRs, after "patient*" and "particip"," that obviously represent what CSRs study. The roots "re$d u c *$ ", that included the terms "reduce(s)," "reducing" and "reduction(s)" and "disab*," that included the terms "disabilities," "disability," "disabled" and "disabling," might represent the researchers' perspective that, generally, aims to reduce or improve "the lack of ability" through the evaluation of rehabilitation interventions effect.

While there is not a single "official" rehabilitation definition, the most important definitions are those issued by the WHO and the United Nations, which define rehabilitation as " $[\ldots]$ a set of interventions designed to optimize functioning and reduce disability in individuals with health conditions in interaction with their environment." 19 In this definition, we can find all the terms identified in our analysis, except for "process". This opens an important 


\section{COPYRIGHT $^{(\odot)} 2020$ EDIZIONI MINERVA MEDICA}

ARIENTI

TERMINOLOGICAL ANALYSIS OF REHABILITATION DEFINITIONS

discussion on what rehabilitation is: a "set of interventions" without a specific interaction between them, or a "process" that combines together a "set of interventions" with a specific order following a project 20 to achieve a predefined result. In the literature, the term "process" has already been used to define rehabilitation..$^{21}$ Derick Wade recently wrote a special edition dedicated to rehabilitation, in which he defined it as "a person-centered process, with treatment tailored to the individual patient's needs and, importantly, personalized monitoring of changes associated with intervention, with further changes in goals and actions if needed. "'22

Therefore, the definition of rehabilitation should include all the identified terms, as they represent the opinion of all the main rehabilitation audiences: consumers, clinicians and researchers. Overall "proces*" should be used as a key element that might change the concept of the rehabilitation definition from a focus on a set (sum) of single interventions to a focus on their interactions which is different from a simple additional effect in the clinical context.23, 24 To improve the effectiveness of rehabilitation interventions, the structure of a rehabilitation definition for research purposes might follow the structure of the research question that represents the starting point for the research studies. ${ }^{25}$ Usually, it guides the definition of the population, interventions and outcomes; consequently, this influences the development of the right study design to answer the question of interest. ${ }^{26}$ This concept could be applied to the rehabilitation definition that might be developed using the structure of the PICO format, the acronym for population, intervention, comparison(s) and outcome.

\section{Limitations of the study}

This study has three main limitations that should be acknowledged. First, there is a disparity between the number of included definitions from the three collections. This arose from the need to be as inclusive as possible, in the effort to extract and convey the key concepts relating to rehabilitation as it is described by the three different audiences. An effort to overcome this limitation was made by deciding to consider the 30 most frequent roots for each collection and analyzing the collection both separately and merged. Furthermore, the frequency of roots present in each collection was expressed as per thousand to mitigate, albeit not completely compensate, the above reported differences. The second limitation, mainly due to time and resources constraints, is the lack of a more complex semantic analysis. That might have brought to light relationships between words and concepts and further deepening the understanding of the various meanings that the term "rehabilitation" has for different audiences. Finally, the definitions considered for CSRs were in fact the descriptions of the interventions: while the two are comparable, they are not exactly the same and this could partly explain some of the differences found among the collections.

\section{Conclusions}

Our findings suggest that it is important that any definition of rehabilitation for research purposes includes all the terms identified focusing on the concept of "process *" and acknowledges interaction between the main elements of rehabilitation. These main elements should encompass the concepts of set of interventions, function and functioning, disability, person, health, optimization and environment. We also suggest to use the structure of the PICO format to develop the definition, in which "P" can be identified as the persons who usually utilize rehabilitation services (i.e. with disabling health conditions), "I" as a specific process focused on functioning and "O" can be identified with the ICF framework. This definition might improve the evaluation of the effectiveness of rehabilitation and its applicability in the clinical context.

\section{References}

1. Wade D. Rehabilitation - a new approach. Overview and Part One: the problems. Clin Rehabil 2015;29:1041-50.

2. Wade DT, de Jong BA. Recent advances in rehabilitation. BMJ 2000;320:1385-8.

3. Negrini S. Evidence in Rehabilitation Medicine: Between Facts and Prejudices. Am J Phys Med Rehabil 2019;98:88-96.

4. Kamioka H, Kawamura Y, Tsutani K, Maeda M, Hayasaka S, Okuizum $\mathrm{H}$, et al. A checklist to assess the quality of reports on spa therapy and balneotherapy trials was developed using the Delphi consensus method: the SPAC checklist. Complement Ther Med 2013;21:324-32.

5. Smith CA, Zaslawski CJ, Cochrane S, Zhu X, Zheng Z, Loyeung B, et al. Reliability of the NICMAN Scale: An Instrument to Assess the Quality of Acupuncture Administered in Clinical Trials. Evid Based Complement Alternat Med 2017;2017:5694083.

6. Philippou YA, Jung JH, Steggall MJ, O’Driscoll ST, Bakker CJ, Bodie $\mathrm{JA}$, et al. Penile rehabilitation for postprostatectomy erectile dysfunction. Cochrane Database Syst Rev 2018;10:CD012414.

7. Rauch A, Negrini S, Cieza A. Toward Strengthening Rehabilitation in Health Systems: Methods Used to Develop a WHO Package of Rehabilitation Interventions. Arch Phys Med Rehabil 2019;100:2205-11.

8. Cochrane Rehabilitation Advisory Board. 2020 [Internet]. Available from: https://rehabilitation.cochrane.org/about-and-contacts/advisoryboard [cited 2020, Aug 31]

9. Cochrane Rehabilitation Executive committee. 2020 [Internet]. Available from: https://rehabilitation.cochrane.org/about-us/executive-committee [cited 2020, Aug 31]

10. Levack WM, Rathore FA, Pollet J, Negrini S. One in 11 Cochrane Reviews Are on Rehabilitation Interventions, According to Pragmatic In- 


\section{COPYRIGHT $^{\circledR} 2020$ EDIZIONI MINERVA MEDICA}

clusion Criteria Developed by Cochrane Rehabilitation. Arch Phys Med Rehabil 2019;100:1492-8.

11. Negrini S, Minozzi S, Taricco M, Ziliani V, Zaina F. A systematic review of physical and rehabilitation medicine topics as developed by the Cochrane Collaboration. Eura Medicophys 2007;43:381-90.

12. Cowie A, Buckley J, Doherty P, Furze G, Hayward J, Hinton S, et al.; British Association for Cardiovascular Prevention and Rehabilitation (BACPR). Standards and core components for cardiovascular disease prevention and rehabilitation. Heart 2019;105:510-5.

13. Piepoli MF, Binno $S$, Corrà U, Seferovic $P$, Conraads V, Jaarsma $T$, et al.; Committee on Exercise Physiology \& Training of the Heart Failure Association of the ESC. ExtraHF survey: the first European survey on implementation of exercise training in heart failure patients. Eur J Heart Fail 2015;17:631-8.

14. Rauch A, Cieza A, Stucki G. How to apply the International Classification of Functioning, Disability and Health (ICF) for rehabilitation management in clinical practice. Eur J Phys Rehabil Med 2008;44:329-42.

15. World Health Organization. International classification of impairments, disabilities, and handicaps: a manual of classification relating to the consequences of disease. Geneva, Switzerland: WHO; 1980. p.205.

16. Cambridge University Press. Cambridge Dictionary; 2020 [Internet]. Available from: https://dictionary.cambridge.org/it/dizionario/inglese/ process [cited 2020, Aug 31]

17. Meyer T, Gutenbrunner C, Bickenbach J, Cieza A, Melvin J, Stucki G. Towards a conceptual description of rehabilitation as a health strategy. J Rehabil Med 2011;43:765-9.
18. European Physical and Rehabilitation Medicine Bodies Alliance. White Book on Physical and Rehabilitation Medicine in Europe. Introductions, Executive Summary, and Methodology. Eur J Phys Rehabil Med 2018;54:125-55

19. World Health Organization. Rehabilitation: key for health in the $21 \mathrm{st}$ century; 2017. [Internet]. Available from: https://www.who.int/disabilities/care/KeyForHealth21stCentury.pdf?ua=1 [cited 2020, Sep 16]

20. Zampolini M, Stucki G, Giustini A, Negrini S. The individual rehabilitation project: a model to strengthen clinical rehabilitation in health systems worldwide. Eur J Phys Rehabil Med 2020;56:1-4.

21. Wade D. Rehabilitation - a new approach. Part four: a new paradigm, and its implications. Clin Rehabil 2016;30:109-18.

22. Wade DT. What is rehabilitation? An empirical investigation leading to an evidence-based description. Clin Rehabil 2020;34:571-83.

23. Negrini S, Arienti C, Kiekens C. Usual care: the big but unmanaged problem of rehabilitation evidence. Lancet 2020;395:337.

24. Negrini S, Arienti C, Pollet J, Engkasan JP, Francisco GE, Frontera WR, et al.; REREP study participants. Clinical replicability of rehabilitation interventions in randomized controlled trials reported in main journals is inadequate. J Clin Epidemiol 2019;114:108-17.

25. Rios LP, Ye C, Thabane L. Association between framing of the research question using the PICOT format and reporting quality of randomized controlled trials. BMC Med Res Methodol 2010;10:11.

26. Sackett DL, Wennberg JE. Choosing the best research design for each question. BMJ 1997;315:1636.

Conflicts of interest.-The authors certify that there is no conflict of interest with any financial organization regarding the material discussed in the manuscript Authors' contributions. - All the authors conceived the paper and its methodology, corrected the manuscript and contributed to the discussion. Chiara Arienti drafted the paper with contributions of Stefano G. Lazzarini and Michele Patrini; Stefano G. Lazzarini and Michele Patrini collected the data; Stefano Negrini, Alex Pollock and Aydan Oral reviewed the draft and helped in the data analysis. All authors read and approved the final version of the manuscript. Acknowledgements.-The authors acknowledge Jaber Firas for the linguistic review of the paper.

History.-Article first published online: October 5, 2020. - Manuscript accepted: September 14, 2020. - Manuscript received: September 8, 2020.

Supplementary data.-For supplementary materials, please see the HTML version of this article at www.minervamedica.it 